\title{
Johnson type bounds for mixed dimension subspace codes
}

\author{
Thomas Honold* \\ ZJU-UIUC Institute \\ International Campus of Zhejiang University \\ Haining, China \\ honold@zju.edu.cn \\ Michael Kiermaier Sascha Kurz ${ }^{\dagger}$ \\ Department of Mathematics \\ University of Bayreuth \\ Bayreuth, Germany \\ \{michael.kiermaier, sascha.kurz\}@uni-bayreuth.de
}

Submitted: Sep 17, 2018; Accepted: Aug 12, 2019; Published: Aug 30, 2019

(C) The authors. Released under the CC BY license (International 4.0).

\begin{abstract}
Subspace codes, i.e., sets of subspaces of $\mathbb{F}_{q}^{v}$ relative to the subspace distance, are applied in random linear network coding. Here we give improved upper bounds for their cardinalities based on the Johnson bound for constant dimension codes.
\end{abstract}

Mathematics Subject Classifications: 05B25,51E20;51E14,51E23

\section{Introduction}

Driven by applications in random linear network coding, the field of subspace coding has sparked a lot of interest recently among both engineers and mathematicians. Various upper bounds on the size of a subspace code have been developed. In the special case of constant dimension codes, the Johnson bound stands out since in general it represents (or, when combined with further knowledge for particular parameters, leads to) the sharpest known bound. In this article we will investigate the Johnson bound for applicability in the case of general mixed dimension subspace codes.

\footnotetext{
*Supported by the National Natural Science Foundation of China under Grant 61571006 .

†Supported supported in part by the grant KU 2430/3-1 - Integer Linear Programming Models for Subspace Codes and Finite Geometry - from the German Research Foundation.
} 
Let $\mathbb{F}_{q}$ be the finite field with $q$ elements, where $q>1$ is a prime power. For $v \geqslant 1$ we denote by $\mathbb{F}_{q}^{v}$ the $v$-dimensional standard vector space over $\mathbb{F}_{q}$. The set of all subspaces of $\mathbb{F}_{q}^{v}$, ordered by the incidence relation $\subseteq$, is called $(v-1)$-dimensional projective geometry over $\mathbb{F}_{q}$ and denoted by $\mathrm{PG}\left(v-1, \mathbb{F}_{q}\right)$ or $\mathrm{PG}\left(\mathbb{F}_{q}^{v}\right)$. It forms a finite modular geometric lattice with meet $X \wedge Y=X \cap Y$, join $X \vee Y=X+Y$, and rank function $X \mapsto \operatorname{dim}(X)$. We will use the term $k$-subspace to denote a $k$-dimensional (vector) subspace of $\mathbb{F}_{q}^{v}$. Using geometric terminology, we also speak of points, lines, planes, and solids for 1-, 2-, 3-, and 4-subspaces, respectively; $(v-1)$-subspaces are also called hyperplanes. The set of all $k$-subspaces of $V=\mathbb{F}_{q}^{v}$ will be denoted by $\left[\begin{array}{c}V \\ k\end{array}\right]$. Its cardinality is given by the Gaussian binomial coefficient

$$
\left[\begin{array}{l}
v \\
k
\end{array}\right]_{q}:= \begin{cases}\frac{\left(q^{v}-1\right)\left(q^{v-1}-1\right) \cdots\left(q^{v-k+1}-1\right)}{\left(q^{k}-1\right)\left(q^{k-1}-1\right) \cdots(q-1)} & \text { if } 0 \leqslant k \leqslant v \\
0 & \text { otherwise. }\end{cases}
$$

For applications in network coding the relevant metric is given by the subspace distance $d_{S}(X, Y):=\operatorname{dim}(X+Y)-\operatorname{dim}(X \cap Y)=2 \operatorname{dim}(X+Y)-\operatorname{dim}(X)-\operatorname{dim}(Y)=\operatorname{dim}(X)+$ $\operatorname{dim}(Y)-2 \operatorname{dim}(X \cap Y)$, which can also be seen as the graph-theoretic distance in the Hasse diagram of $\operatorname{PG}\left(v-1, \mathbb{F}_{q}\right)$. A set $\mathcal{C}$ of subspaces of $\mathbb{F}_{q}^{v}$ is called a subspace code. The minimum (subspace) distance of $\mathcal{C}$ is given by $d=\min \left\{d_{S}(X, Y): X, Y \in \mathcal{C}, X \neq\right.$ $Y\}$. If all elements of $\mathcal{C}$ have the same dimension, we call $\mathcal{C}$ a constant dimension code. By $\mathrm{A}_{q}(v, d)$ we denote the maximum possible cardinality of a subspace code in $\mathbb{F}_{q}^{v}$ with minimum distance at least $d$. Analogously, $\mathrm{A}_{q}(v, d ; k)$ denotes the maximum cardinality of a constant dimension code with codewords of dimension $k$. Like in the classical case of codes in the Hamming metric, the determination of the exact value or bounds for $\mathrm{A}_{q}(v, d)$ and $\mathrm{A}_{q}(v, d ; k)$ is an important problem. In this paper we will present some improved upper bounds. For a broader background we refer to $[6,8]$ and for the latest numerical bounds to the online tables at http://subspacecodes.uni-bayreuth. de [12].

Constant dimension codes with $d=2 k$ are called partial $k$-spreads. A vector space partition $\mathcal{P}$ of $\mathbb{F}_{q}^{v}$ is a set of nonzero subspaces such that each point of $\mathbb{F}_{q}^{v}$ is contained in exactly one element of $\mathcal{P}$. If $\mathcal{P}$ consists of $m_{i}$ subspaces of dimension $i$ for $1 \leqslant i \leqslant v$, then we say that $\mathcal{P}$ has type $1^{m_{1}} 2^{m_{2}} \ldots v^{m_{v}}$.

The remaining part of this paper is organized as follows. In Section 2 we review known upper bounds for subspace codes. Johnson type bounds for constant dimension codes are presented in Section 3, before the underlying concept is generalized to the mixed dimension case in Section 4. Analytic upper bounds for $A_{q}(v, v-4)$ and $A_{q}(8,3)$ are then determined in Section 5. We draw a brief conclusion in Section 6.

\section{Known upper bounds for mixed dimension codes}

As mentioned in the introduction, our main focus is on the determination of $A_{q}(v, d)$. In that process we will often encounter codes with a restricted set $K \subseteq\{0,1, \ldots, v\}$ of possible dimensions, so that we will also consider upper bounds for $A_{q}(v, d ; K)$, the maximum cardinality of a subspace code in $\mathbb{F}_{q}^{v}$ with minimum distance at least $d$ and all 
codeword dimensions contained in $K$. Especially, $A_{q}(v, d ;\{k\})=A_{q}(v, d ; k)$. The most obvious facts about the numbers $A_{q}(v, d ; K)$ are summarized in [18, Lemma 2.3]: Clearly we have monotonicity in $d$ and $K$, i.e., $A_{q}(v, d ; K) \geqslant A_{q}\left(v, d^{\prime} ; K\right)$ for $1 \leqslant d \leqslant d^{\prime} \leqslant v$ and $A_{q}(v, d ; K) \leqslant A_{q}\left(v, d ; K^{\prime}\right)$ for $K \subseteq K^{\prime} \subseteq\{0, \ldots, v\}$. By decomposing codes we obtain $A_{q}\left(v, d ; K \cup K^{\prime}\right) \leqslant A_{q}(v, d ; K)+A_{q}\left(v, d ; K^{\prime}\right)$. Considering the dual subspace code $\mathcal{C}^{\perp}=\left\{X^{\perp} ; X \in \mathcal{C}\right\}$ of $\mathcal{C}$ gives $A_{q}(v, d, K)=A_{q}(v, d, v-K)$ with $v-K=\{v-k: k \in K\}$. Subspace distance $d=1$ permits to choose all subspaces, i.e., $A_{q}(v, 1 ; K)=\sum_{i \in K}\left[\begin{array}{l}v \\ i\end{array}\right]_{q}$. For subspace distance $d=2$ the optimal codes in the unrestricted mixed dimension case are classified in [18, Theorem 3.4] with cardinalities

$$
A_{q}(v, 2)=\sum_{i \equiv\lfloor v / 2\rfloor(\bmod 2)}\left[\begin{array}{l}
v \\
i
\end{array}\right]_{q}
$$

if $v$ is even and

$$
A_{q}(v, 2)=\sum_{i \equiv 0}\left[\begin{array}{l}
v \\
i
\end{array}\right]_{q}=\sum_{i \equiv 1}\left[\begin{array}{l}
v \\
i
\end{array}\right]_{q}
$$

if $v$ is odd. For subspace distance $d=v$ we have $A_{q}(v, v)=2$ if $v$ is odd and $A_{q}(v, v)=$ $A_{q}(v, v ; k)=q^{k}+1$ if $v$ is even, see [18, Theorem 3.1]. Also subspace distance $v-1$ has been resolved completely, see [18, Theorem 3.2]. If $v=2 m$ is even then $A_{q}(v, v-1)=$ $A_{q}(v, v ; m)=q^{m}+1$, and if $v=2 m+1 \geqslant 5$ is odd then $A_{q}(v, v-1)=A_{q}(v, v-1 ; m)=$ $q^{m+1}+1$. Note that $A_{q}(3,2)=q^{2}+q+2>q^{2}+1$. So, in the following we can always assume $v \geqslant 5$ and $3 \leqslant d \leqslant v-2$. For subspace distance $d=v-2$ there is so far only partial information, see [18, Theorem 3.3]. If $v=2 m \geqslant 8$ is even, then $A_{q}(v, v-2)=A_{q}(v, v-2 ; m)$ with $q^{2 m}+1 \leqslant A_{q}(v, v-2 ; m) \leqslant\left(q^{m}+1\right)^{2}$. Moreover, $A_{q}(4,2)=q^{4}+q^{3}+2 q^{2}+q+3$ for all $q, A_{2}(6,4)=A_{2}(6,4 ; 3)=77, q^{6}+2 q^{2}+2 q+1 \leqslant$ $A_{q}(6,4) \leqslant\left(q^{3}+1\right)^{2}$ for $q \geqslant 3$, see [16], and $A_{2}(8,6)=A_{2}(8,6 ; 4)=257$ [9]. The 8 isomorphism types of all latter optimal codes have been classified in [15]. If $v=2 m+1 \geqslant 5$ is odd, then $A_{q}(v, v-2) \in\left\{2 q^{m+1}+1,2 q^{m+1}+2\right\}$. Moreover, $A_{q}(5,3)=2 q^{3}+2$ for all $q$ and $A_{2}(7,5)=2 \cdot 2^{4}+2=34$. The 20 isomorphism types of all latter optimal codes have been classified in [17].

Next we consider upper bounds for mixed dimension codes that are applicable for all parameters. Since the minimum subspace distance in a constant dimension code is even, decomposing the code into constant dimension codes gives $A_{q}(v, d) \leqslant \sum_{i=0}^{v} A_{q}(v, 2\lceil d / 2\rceil ; i)$. Observing $A_{q}(v, d ;\{0,1, \ldots,\lceil d / 2\rceil-1\})=A_{q}(v, d ;\{v-\lceil d / 2\rceil+1, \ldots, v-1, v\})=1$, this was slightly tightened to $A_{q}(v, d) \leqslant 2+\sum_{i=\lceil d / 2\rceil}^{v-\lceil d / 2\rceil} A_{q}(v, 2\lceil d / 2\rceil ; i)$ in [18, Theorem 2.5]. There is yet another tiny improvement, which seems to have been unnoticed so far:

Lemma 1. If $\lceil d / 2\rceil$ divides $v$, then $A_{q}(v, d) \leqslant \sum_{i=\lceil d / 2\rceil}^{v-\lceil d / 2\rceil} A_{q}(v, 2\lceil d / 2\rceil ; i)$.

Proof. The constant dimension codes attaining $A_{q}(v, 2\lceil d / 2\rceil ;\lceil d / 2\rceil)$ are spreads, which cover each point exactly once and hence have distance $<d$ to all subspaces of dimension $k \in\{0,1, \ldots,\lceil d / 2\rceil-1\}$ (and similarly for $i=v-\lceil d / 2\rceil$ ). 
Let us remark that this lemma gives $A_{2}(6,3) \leqslant 119$. By extending the known five isomorphism types of constant dimension codes attaining $A_{2}(6,4 ; 3)=77$ one can reduce this bound by 1 to $A_{2}(6,3) \leqslant 118$, see [18, Section 4.2]. For the best known bounds on $A_{2}(v, d)$ with $v \leqslant 8$ we refer the reader to [15].

According to [2] the, so far, only successful generalization of the classical bounds to projective space was given by Etzion and Vardy in [7, Theorem 10]. The approach generalizes the sphere-packing bound for constant dimension codes facing the fact that the spheres have different sizes. To that end let $B(U, e)$ denote the ball with center $U$ and radius $e$. Those balls around codewords are pairwise disjoint for subspace distance $d=2 e+1$. Denoting the number of $k$-dimensional subspaces contained in $B(U, e)$ with $\operatorname{dim}(U)=i$ by $c(i, k, e)$, we have

$$
c(i, k, e)=\sum_{j=\left\lceil\frac{i+k-e}{2}\right\rceil}^{\min \{k, i\}}\left[\begin{array}{l}
i \\
j
\end{array}\right]_{q}\left[\begin{array}{c}
v-i \\
k-j
\end{array}\right]_{q} q^{(i-j)(k-j)} .
$$

Thus, $A_{q}(v, 2 e+1)$ is at most as large as the target value of the ILP:

$$
\begin{array}{cc}
\max \sum_{i=0}^{v} a_{i} & \text { subject to } \\
a_{i} \leqslant A_{q}(v, 2 e+2 ; i) & \forall 0 \leqslant i \leqslant v \\
\sum_{i=0}^{v} c(i, k, e) \cdot a_{i} \leqslant\left[\begin{array}{l}
v \\
k
\end{array}\right]_{q} & \forall 0 \leqslant k \leqslant v \\
a_{i} \in \mathbb{N} & \forall 0 \leqslant i \leqslant v
\end{array}
$$

Here, the $a_{i}$ denote the number of codewords of dimension $i$. As for each ILP one can consider the LP relaxation, i.e., replacing the integer variables by non-negative real variables, in order to decrease computation times. For this ILP it turns out that the gap between the target value of the ILP and the corresponding LP is quite often smaller than 1. Note that the described sphere-packing approach for even distances is obtained via $A_{q}(v, 2 e+2) \leqslant A_{q}(v, 2 e+1)$, which nevertheless turns out to be the best known upper bound in some cases, see e.g. the bounds for $A_{2}(10,6)$ and $A_{2}(10,5)$ in [12].

As the problem of the determination of $A_{q}(v, d)$ can be naturally formulated as a maximum clique problem, and the Lovász theta bound from semidefinite programming can be applied. Since the problem size is linear in terms of the graph parameters, they are exponential in $v$. However, one can take the acting symmetry group into account in order to drastically decrease the problem size, see [1] for general reduction techniques for invariant semidefinite programs. Obtaining parametric formulas for the reduced SDP formulations is a highly non-trivial task in general, and was achieved for $\vartheta^{\prime}$ of the graph corresponding to $A_{q}(v, d)$ in [2]. The authors report several numerical results for $q=2$ and odd distances. Where they are computed, this gives the best known upper bound in many cases. Using improved upper bounds for constant dimension codes, especially partial spreads, in [10] the authors compute numerical results, also for $q>2$ and even distances. 


\section{Johnson type bounds for constant dimension codes}

One approach to obtain upper bounds for constant dimension codes is to try to generalize upper bounds for binary error-correcting constant weight codes in the Hamming metric, which corresponds to the case $q=1$. Several of the latter have been obtained by Johnson in 1962. The bound [20, Theorem 3], see also [27], has been generalized by Xia and Fu to [28, Theorem 2]. However, the formulation of the bound can be simplified considerably, see [13, Proposition 1], and only applies to partial spreads, i.e., $d=2 k$. While the generalization of [20, Theorem 3] is rather weak, generalizing [20, Inequality (5)] yields a considerably stronger upper bound:

Theorem 2. (Johnson type bound II) [28, Theorem 3], [7, Theorems 4 and 5]

$$
\begin{aligned}
& A_{q}(v, d ; k) \leqslant\left\lfloor\frac{q^{v}-1}{q^{k}-1} A_{q}(v-1, d ; k-1)\right\rfloor \\
& A_{q}(v, d ; k) \leqslant\left\lfloor\frac{q^{v}-1}{q^{v-k}-1} A_{q}(v-1, d ; k)\right\rfloor
\end{aligned}
$$

For the proof of Inequality (2) one considers all codewords containing an arbitrary but fixed point. Since there are at most $A_{q}(v-1, d ; k-1)$ such codewords and the number of points in the ambient space and in a codeword is $\left[\begin{array}{l}v \\ 1\end{array}\right]_{q}$ and $\left[\begin{array}{l}k \\ 1\end{array}\right]_{q}$, respectively, the upper bound follows. Inequality (3) is obtained if one considers codewords contained in a given hyperplane instead. We remark that Inequality (2) and Inequality (3) are equivalent using duality, i.e., $A_{q}(v, d ; k)=A_{q}(v, d ; v-k)$.

Of course, the bounds in Theorem 2 can be applied iteratively. For binary errorcorrecting constant weight codes in the Hamming metric the optimal choice of the corresponding inequalities is unclear, see e.g. [24, Research Problem 17.1], while we have:

Proposition 3. [13, Proposition 2]

For $k \leqslant v / 2$ we have

$$
\left\lfloor\frac{q^{v}-1}{q^{k}-1} A_{q}(v-1, d ; k-1)\right\rfloor \leqslant\left\lfloor\frac{q^{v}-1}{q^{v-k}-1} A_{q}(v-1, d ; k)\right\rfloor,
$$

where equality holds iff $v=2 k$.

So, initially assuming $k \leqslant v / 2$, the optimal choice is to iteratively apply Inequality (2), which results in:

\section{Corollary 4. (Implication of the Johnson type bound II)}

$$
A_{q}(v, d ; k) \leqslant\left\lfloor\frac{q^{v}-1}{q^{k}-1}\left\lfloor\frac{q^{v-1}-1}{q^{k-1}-1}\left\lfloor\ldots\left\lfloor\frac{q^{v-k+d / 2+1}-1}{q^{d / 2+1}-1} A_{q}(v-k+d / 2, d ; d / 2)\right\rfloor \ldots\right\rfloor\right\rfloor\right\rfloor .
$$

We prefer not to insert $A_{q}(v-k+d / 2, d ; d / 2) \leqslant\left\lfloor\frac{q^{v-k+d / 2}-1}{q^{d / 2}-1}\right\rfloor$, since currently much better bounds for partial spreads are available, which we will discuss next. 
In the case $d=2 k$, any two codewords of $\mathcal{C}$ intersect trivially, meaning that each point of $\mathrm{PG}\left(\mathbb{F}_{q}^{v}\right)$ is covered by at most a single codeword. These codes are better known as partial $k$-spreads. If all the points are covered, we have $\# \mathcal{C}=\left[\begin{array}{l}v \\ 1\end{array}\right]_{q} /\left[\begin{array}{l}k \\ 1\end{array}\right]_{q}$ and $\mathcal{C}$ is called a $k$-spread. From the work of Segre in 1964 [26, $\S \mathrm{VI}]$ we know that $k$-spreads exist if and only if $k$ divides $v$. Upper bounds for the size of a partial $k$-spreads are due to Beutelspacher [3] and Drake \& Freeman [5] and date back to 1975 and 1979, respectively. Starting with [22], several recent improvements have been obtained. Currently the tightest upper bounds, besides $k$-spreads, are given by a list of 21 sporadic 1-parametric series and the following two theorems stated in [23]:

Theorem 5. For integers $r \geqslant 1, t \geqslant 2, u \geqslant 0$, and $0 \leqslant z \leqslant\left[\begin{array}{l}r \\ 1\end{array}\right]_{q} / 2$ with $k=\left[\begin{array}{l}r \\ 1\end{array}\right]_{q}+1-$ $z+u>r$ we have $\mathrm{A}_{q}(v, 2 k ; k) \leqslant l q^{k}+1+z(q-1)$, where $l=\frac{q^{v-k}-q^{r}}{q^{k}-1}$ and $v=k t+r$.

Theorem 6. For integers $r \geqslant 1, t \geqslant 2, y \geqslant \max \{r, 2\}, z \geqslant 0$ with $\lambda=q^{y}, y \leqslant k$, $k=\left[\begin{array}{l}r \\ 1\end{array}\right]_{q}+1-z>r, v=k t+r$, and $l=\frac{q^{v-k}-q^{r}}{q^{k}-1}$, we have $\mathrm{A}_{q}(v, 2 k ; k) \leqslant$

$$
l q^{k}+\left\lceil\lambda-\frac{1}{2}-\frac{1}{2} \sqrt{1+4 \lambda(\lambda-(z+y-1)(q-1)-1)}\right\rceil .
$$

The special case $z=0$ in Theorem 5 covers the breakthrough $\mathrm{A}_{q}(k t+r, 2 k ; k)=$ $1+\sum_{s=1}^{t-1} q^{s k+r}$ for $0<r<k$ and $k>\left[\begin{array}{l}r \\ 1\end{array}\right]_{q}$ by Năstase and Sissokho [25] from 2016, which itself covers the result of Beutelspacher. The special case $y=k$ in Theorem 6 covers the result by Drake \& Freeman. A contemporary survey of the best known upper bounds for partial spreads can be found in [19].

All currently known upper bounds for partial spreads can be deduced from the nonexistence of certain divisible codes, see [19]. The set $N$ of all points of the ambient space not contained in any $k$-space of a partial spread corresponds to a projective linear code over $\mathbb{F}_{q}$ of length $n=\# N$ with all codeword Hamming weights divisible by $q^{k-1}$. Recently this idea was generalized to constant dimension codes with $d<2 k$ in [21]. If $\mathcal{C}$ is a set of subspaces with dimensions at least $r$ and such that every point $P$ is contained in at most $j$ subspaces $X \in \mathcal{C}$, then the multiset $N$ of points defined by $P \mapsto j-\#\{X \in \mathcal{C} ; P \in X\}$ corresponds to a possibly non-projective linear code over $\mathbb{F}_{q}$ of length $\# N$ with all codeword Hamming weights divisible by $q^{r-1}$. Let $\left\{a /\left[\begin{array}{l}k \\ 1\end{array}\right]_{q}\right\}_{k}$ denote the maximal $b \in \mathbb{N}$ permitting a $q^{k-1}$-divisible code of length $a-b \cdot\left[\begin{array}{l}k \\ 1\end{array}\right]_{q}$ over $\mathbb{F}_{q}$. With this, Inequality (2) can be tightened (obviously we have $\left\{a /\left[\begin{array}{l}k \\ 1\end{array}\right]_{q}\right\}_{k} \leqslant\left\lfloor a /\left[\begin{array}{l}k \\ 1\end{array}\right]_{q}\right\rfloor$ ) to

$$
A_{q}(v, d ; k) \leqslant\left\{\left[\begin{array}{l}
v \\
1
\end{array}\right]_{q} \cdot A_{q}(v-1, d ; k-1) /\left[\begin{array}{l}
k \\
1
\end{array}\right]_{q}\right\}_{k} .
$$

Using the abbreviation $v^{\prime}=v-k+d / 2$, the iterated application yields $A_{q}(v, d ; k)$

$$
\leqslant\left\{\frac{\left[\begin{array}{l}
v \\
1
\end{array}\right]_{q}}{\left[\begin{array}{l}
k \\
1
\end{array}\right]_{q}} \cdot\left\{\frac{\left[\begin{array}{c}
v-1 \\
1
\end{array}\right]_{q}}{\left[\begin{array}{c}
k-1 \\
1
\end{array}\right]_{q}} \cdot\left\{\cdots\left\{\frac{\left[\begin{array}{c}
v^{\prime}+1 \\
1
\end{array}\right]_{q}}{\left[\begin{array}{c}
d / 2+1 \\
1
\end{array}\right]_{q}} \cdot A_{q}\left(v^{\prime}, d ; d / 2\right)\right\}_{d / 2+1} \ldots\right\}_{k-2}\right\}_{k-1}\right\}_{k}
$$


which is the tightened version of Corollary 4 .

While the question whether a projective $q^{r}$-divisible linear code over $\mathbb{F}_{q}$ of length $n$ exists, is unsolved in general, this problem has been solved in the non-projective case via an efficient algorithm, see [21, Theorem 4] and [21, Algorithm 1], i.e., $\left\{a /\left[\begin{array}{l}k \\ 1\end{array}\right]_{q}\right\}_{k}$ can be computed efficiently. Results from the theory of $q^{r}$-divisible linear codes over $\mathbb{F}_{q}$ are exemplarily applied in Lemma 11.

We remark that Inequality (4) combined with the best known upper bounds for partial spreads yields the best known upper bounds for constant dimension codes except for $\mathrm{A}_{2}(6,4 ; 3)=77<81[16]$ and $\mathrm{A}_{2}(8,6 ; 4)=257 \leqslant 272<289[9,14]$. The mentioned improvements are based on extensive integer linear programming computations. In contrast to that, the improvements in this article are based on self-contained theoretical arguments and do not need any huge computations.

\section{Johnson type bounds for mixed dimension codes}

Since Theorem 2 (and its refinement based on $q^{r}$-divisible codes) is that competitive for constant dimension codes, it seems quite natural to investigate the underlying idea also in the mixed dimension case. As the number of points in subspaces of different dimensions is different, we have to take the precise dimension distribution of those codewords that contain a specific point into account. To that end let $F_{q}(v, d)$ be the set of $(v+1)$-tuples $b=\left(b_{0}, \ldots, b_{v}\right) \in \mathbb{N}^{v+1}$ such that there exists a mixed dimension code $\mathcal{C}$ in $\mathbb{F}_{q}^{v}$ with minimum distance at least $d$ and dimension distribution $b$. Note that our numbering starts from 0 . Transferring the idea of the Johnson bound we end up with the following integer linear programming (ILP) formulation

Proposition 7. For all $v \geqslant 2$ and $1 \leqslant d \leqslant v$ we have

$$
\begin{aligned}
A_{q}(v, d) \leqslant \max \sum_{i=0}^{v} a_{i} & \text { subject to } \\
\sum_{b \in F_{q}(v-1, d)} b_{i-1} x_{b}=\left[\begin{array}{l}
i \\
1
\end{array}\right]_{q} a_{i} & \forall 1 \leqslant i \leqslant v \\
\sum_{b \in F_{q}(v-1, d)} x_{b}=\left[\begin{array}{l}
v \\
1
\end{array}\right]_{q} & \\
a_{0} \leqslant 1 & \\
A_{q}(v, d ; i) a_{0}+a_{i} \leqslant A_{q}(v, d ; i) & \forall 1 \leqslant i \leqslant d-1 \\
x_{b} \in \mathbb{N} & \forall b \in F_{q}(v-1, d) \\
a_{i} \in \mathbb{N} & \forall 1 \leqslant i \leqslant v
\end{aligned}
$$

Proof. Let $\mathcal{C}$ be a subspace code in $\mathbb{F}_{q}^{v}$ with minimum subspace distance at least $d$ whose cardinality attains $A_{q}(v, d)$. By $a_{i} \in \mathbb{N}$ we denote the number of $i$-dimensional codewords for $0 \leqslant i \leqslant v$, i.e., vector $a$ is the dimension distribution of $\mathcal{C}$. For every point $P$ of $\mathbb{F}_{q}^{v}$ let 
$\mathcal{C}_{P}$ be $\{C \in \mathcal{C}: P \leqslant C\}$ modulo $P$ and $b_{P} \in \mathbb{N}^{v}$ be the dimension distribution of $\mathcal{C}_{P}$. Thus $b_{P} \in F_{q}(v-1, d)$. By $x_{b} \in \mathbb{N}$ we count the number of points $p$ such that $b=b_{P}$. Since each $i$-dimensional codeword of $\mathcal{C}$ contains $\left[\begin{array}{l}i \\ 1\end{array}\right]_{q}$ points we have $\sum_{b \in F_{q}(v-1, d)} b_{i-1} x_{b}=\left[\begin{array}{c}i \\ 1\end{array}\right]_{q} a_{i}$ for all $1 \leqslant i \leqslant v$. Since every $b_{P}$ is counted exactly once, we have $\sum_{b \in F_{q}(v-1, d)} x_{b}=\left[\begin{array}{l}v \\ 1\end{array}\right]_{q}$. Of course $\mathcal{C}$ can contain at most one 0 -dimensional codeword. Since $a_{0}$ is not coupled with the $x_{b}$-variables, we use the fact that $\mathcal{C}$ can not contain both a 0 - and an $i$-dimensional codeword for $1 \leqslant i \leqslant d-1$. This can be modeled as $A_{q}(v, d ; i) a_{0}+a_{i} \leqslant A_{q}(v, d ; i)$, i.e., if $a_{0}=1$ then the inequality reads $a_{i} \leqslant 0$ and if $a_{0}=0$ then the inequality is equivalent to $a_{i} \leqslant A_{q}(v, d ; i)$, which is also valid.

We remark that the hard-to-compute values $A_{q}(v, d ; i)$, occurring as coefficients of inequalities in the above ILP, may be replaced by any upper bound on $A_{q}(v, d ; i)$ and the set $F_{q}(v-1, d)$ may be also replaced by any superset, which of course may weaken the resulting upper bound. Of course, further inequalities like e.g. $\sum_{i \in K} a_{i} \leqslant A_{q}(v, d ; K)$ for some $K \subseteq\{0, \ldots, v\}$ may be added.

Having Proposition 3 at hand, it is obvious that ILP formulation (5) can be further improved by also taking Inequality (3) into account. However, instead of considering subcodes in hyperplanes we use duality in order to assume $\sum_{i=0}^{\lfloor v / 2\rfloor} a_{i} \geqslant \sum_{\lceil v / 2\rceil}^{v} a_{i}$, which also allows us to eliminate variables. As a shortcut, we use $m=\lfloor v / 2\rfloor$ in the following and denote by $\bar{F}_{q}(v-1, d)$ the set of $m$-tuples $b=\left(b_{0}, \ldots, b_{m-1}\right) \in \mathbb{N}^{m}$ such that there exists a subspace code $\mathcal{C}$ in $\mathbb{F}_{q}^{v-1}$ with minimum subspace distance, at least, $d$, codewords with dimensions in $\{0, \ldots, m-1\}$, and dimension distribution $b$. With this we can directly reformulate Proposition 7 to:

Proposition 8. For all $v \geqslant 2$ and $1 \leqslant d \leqslant v$ we have

$$
\begin{aligned}
& A_{q}(v, d) \leqslant \max t(a) \quad \text { subject to } \\
& \sum_{b \in \bar{F}_{q}(v-1, d)} b_{i-1} x_{b}=\left[\begin{array}{l}
i \\
1
\end{array}\right]_{q} a_{i} \quad \forall 1 \leqslant i \leqslant m \\
& \sum_{b \in \bar{F}_{q}(v-1, d)} x_{b}=\left[\begin{array}{l}
v \\
1
\end{array}\right]_{q} \\
& a_{0} \leqslant 1 \\
& A_{q}(v, d ; i) a_{0}+a_{i} \leqslant A_{q}(v, d ; i) \quad \forall 1 \leqslant i \leqslant \min \{d-1, m\} \\
& x_{b} \in \mathbb{N} \quad \forall b \in \bar{F}_{q}(v-1, d) \\
& a_{i} \in \mathbb{N} \quad \forall 0 \leqslant i \leqslant m,
\end{aligned}
$$

where $m=\lfloor v / 2\rfloor$ and $t(a)=2 \sum_{i=0}^{m} a_{i}$ if $v-1$ is even and by $t(a)=a_{m}+2 \sum_{i=0}^{m-1} a_{i}$ otherwise.

Now let us compute the contribution of a point $P$ with dimension distribution $b_{P}$ of 
the corresponding code $\mathcal{C}_{P}$. For $b=b_{P} \in \mathbb{N}^{m}$ and $m=\lfloor v / 2\rfloor$ we set

$$
\Gamma_{v}(b)=\left\{\begin{array}{r}
2 \sum_{i=0}^{m-1} \frac{b_{i}}{\left[\begin{array}{c}
i+1 \\
1
\end{array}\right]_{q}}: v \equiv 1 \quad(\bmod 2), \\
\frac{b_{m-1}}{\left[\begin{array}{c}
m \\
1
\end{array}\right]_{q}}+2 \sum_{i=0}^{m-2} \frac{b_{i}}{\left[\begin{array}{c}
i+1 \\
1
\end{array}\right]_{q}} \quad: \quad v \equiv 0 \quad(\bmod 2) .
\end{array}\right.
$$

and call $\Gamma_{v}(b)$ score of $b$. With this we have

$$
t(a)=\sum_{b} x_{b} \Gamma_{v}(b) \leqslant\left[\begin{array}{l}
v \\
1
\end{array}\right]_{q} \cdot \max _{b} \Gamma_{v}(b)
$$

In other words, we express the target function in terms of the $x_{b}$ and ignore all constraints on the $a_{i}$, giving an easy upper bound.

Even for small parameters $v$ and $d$ the sets $\bar{F}_{q}(v-1, d)$ can become quite large, so that we introduce another ILP variant. To that end let us write $c^{\prime} \leqslant c$ for two vectors $c^{\prime}, c \in \mathbb{R}^{n}$ iff $c_{i}^{\prime} \leqslant c_{i}$ for all $1 \leqslant i \leqslant n$, where the integer $n$ will be always clear from the context. Of course we have $\Gamma_{v}\left(c^{\prime}\right) \leqslant \Gamma_{v}(c)$. Let $\bar{F}_{q}(v-1, d) \subset \mathcal{F}$ for some set $\mathcal{F}$. We call an element $f$ of $\mathcal{F}$ maximal if there does not exist an element $f^{\prime} \in \mathcal{F}$ with $f^{\prime} \geqslant f$ and $f^{\prime} \neq f$. If $\overline{\mathcal{F}}$ contains all maximal elements from $\mathcal{F}$ then we can restrict to $b \in \overline{\mathcal{F}}$ in Proposition 8 if we replace $\sum_{b \in \mathcal{F}} b_{i-1} x_{b}=\left[\begin{array}{l}i \\ 1\end{array}\right]_{q} a_{i}$ by $\sum_{b \in \overline{\mathcal{F}}} b_{i-1} x_{b} \geqslant\left[\begin{array}{l}i \\ 1\end{array}\right]_{q} a_{i}$.

Combining both ideas gives:

Proposition 9. Let $v \geqslant 2$ and $1 \leqslant d \leqslant v$ be integers, $\omega \in \mathbb{R}_{\text {ge0 }}$, and $m=\lfloor v / 2\rfloor$. If $\mathcal{F} \subseteq \mathbb{N}^{m}$ such that for all $f^{\prime} \in \bar{F}_{q}(v-1, d)$ either there exists an element $f \in \mathcal{F}$ with $f \geqslant f^{\prime}$ or $\Gamma_{v}\left(f^{\prime}\right) \leqslant \omega$, then we have

$$
\begin{array}{cl}
A_{q}(v, d) \leqslant \max \omega z+t(v) & \text { subject to } \\
\sum_{b \in \mathcal{F}} b_{i-1} x_{b} \geqslant\left[\begin{array}{l}
i \\
1
\end{array}\right]_{q} a_{i} & \forall 1 \leqslant i \leqslant m \\
z+\sum_{b \in \mathcal{F}} x_{b}=\left[\begin{array}{l}
v \\
1
\end{array}\right]_{q} & \\
A_{q}(v, d ; i) a_{0}+a_{i} \leqslant A_{q}(v, d ; i) & \forall 1 \leqslant i \leqslant \min \{d-1, m\} \\
a_{i} \leqslant A_{q}(v, d ; i) & \forall \min \{d-1, m\}+1 \leqslant i \leqslant m \\
x_{b} \in \mathbb{N} & \forall b \in \mathcal{F} \\
z \in \mathbb{N}, &
\end{array}
$$

where $t(a)$ is defined as in Proposition 8.

Proof. We extend the ILP model from Proposition 8 by counting $b_{P}$ either in $x_{b}$ where $b_{P} \leqslant b$ and $b \in \mathcal{F}$ or in the new auxiliary variable $z$ (then $\Gamma_{v}\left(b_{P}\right) \leqslant \omega$ ). The interpretation of the $a_{i}$ changes slightly if $z>0$ since some contributions of the $b_{P}$ are hidden in $z$. 
Note that we can add the restrictions $a_{i} \in \mathbb{N}$ if $z=0$, i.e., the $a_{i}$ keep their meaning as the dimension distribution of the code $\mathcal{C}$.

In the following we will mostly use the ILP formulation (9) in order to compute improved upper bounds for $A_{q}(v, d)$. It remains to provide an algorithm to compute a feasible set $\mathcal{F}$. For all $b \in \bar{F}_{q}(v-1, d)$ we obviously have $0 \leqslant b_{i} \leqslant A_{q}(v-1, d ; i)$, so that there is only a finite number of possibilities. In order to check whether $b \in \bar{F}_{q}(v-1, d)$ we slightly modify the ILP formulation (9) by setting $z=0$, replacing $v$ by $v-1$, adding the constraints $a_{i} \geqslant b_{i}$ for all $i \neq j$, and replacing the target function by $a_{j}$, where $j$ can be chosen freely. If there is no solution, then $b \notin \bar{F}_{q}(v-1, d)$. Otherwise the solution vector $a$ can be added to $\mathcal{F}$ and all $b^{\prime} \leqslant a$ do not need to be considered any more. Moreover, all vectors where $a_{j}$ is replaced by a larger number in $a$ cannot be contained in $\bar{F}_{q}(v-1, d)$. This gives a recursive algorithm, which works in principle. For larger parameters, it will become computationally infeasible. However, by a mixture between theoretical reasoning and (I)LP computations we will be able to determine suitable sets $\mathcal{F}$ for many parameters. In the determination of $\mathcal{F}$ we will speak of maximal patterns.

We give a concrete numerical example:

Lemma 10. $A_{2}(10,5) \leqslant 48104$

Proof. Let $\mathcal{C}$ be a subspace code of $\mathbb{F}_{2}^{10}$ with minimum subspace distance $d=5$, and let $a_{i}$ denote the number of $i$-dimensional codewords. W.l.o.g. we assume $a_{0}+a_{1}+a_{2}+a_{3}+a_{4} \geqslant$ $a_{6}+a_{7}+a_{8}+a_{9}+a_{10}$, so that $\# \mathcal{C} \leqslant a_{5}+2 \sum_{i=0}^{4} a_{i}$. If $a_{0}+a_{1} \geqslant 1$, then $a_{0}+a_{1}=1$ and $\# \mathcal{C} \leqslant 2+2 a_{4}+a_{5} \leqslant 2+2 \cdot 4977+38148=48104$ due to $A_{2}(10,5 ; 4) \leqslant 4977$ and $A_{2}(10,5 ; 5) \leqslant 38148$. Thus, we assume $a_{0}=a_{1}=0$ in the following.

Next we consider the possible maximal patterns of codewords through a point, i.e., in $\mathbb{F}_{2}^{9}$ we consider sets of codewords with dimension in $\{1,2,3,4\}$ and minimum distance at least 5 . Since $A_{2}(9,6 ; 3)=73$ and $A_{2}(9,6 ; 4) \leqslant 1156$ the maximal patterns are below $1^{1} 4^{1156}, 3^{73} 4^{1156}$, or patterns of the form $2^{1} 3^{x_{3}} 4^{x_{4}}$. So, let us determine bounds for $x_{3}$ and $x_{4}$. In $\mathbb{F}_{2}^{8}$ is suffices to consider the patterns $1^{1}$ and $2^{1} 3^{34}$ since $A_{2}(8,5 ; 1)=A_{2}(8,5 ; 2)=1$ and $A_{2}(8,5 ; 3)=34$. Only pattern $2^{1} 3^{34}$ contributes to $x_{3}$ or $x_{4}$. Since a line is present, we need at least 3 times pattern $1^{1}$, so that at most $\left[\begin{array}{l}9 \\ 1\end{array}\right]_{2}-3=508$ points in $\mathbb{F}_{2}^{9}$ can have pattern $2^{1} 3^{34}$. Thus we have $x_{3} \leqslant\left\lfloor 508 /\left[\begin{array}{l}3 \\ 1\end{array}\right]_{2}\right\rfloor=72$ and $x_{4} \leqslant\left\lfloor 508 \cdot 34 /\left[\begin{array}{l}4 \\ 1\end{array}\right]_{2}\right\rfloor=1151$.

Finally, let

$$
\mathcal{F}=\{(0,1,0,0,1156),(0,0,0,73,1156),(0,0,1,72,1151)\}
$$

and apply the ILP of Proposition 9 with $z=0$. This gives $\# \mathcal{C} \leqslant 48104$. An optimal solution is given by $a_{3}=3, a_{4}=4977$ and $b_{5}=38144$, where the second pattern is chosen 999 and the third pattern is chosen 24 times.

Note that the scores $\Gamma_{10}$ of the three patterns in $\mathcal{F}$ are less than 37.95699, 47.023656, and 47.014747, respectively, so that Inequality (8) would give $\# \mathcal{C} \leqslant\lfloor 48105.3\rfloor=48105$, i.e., the solution of the ILP slightly pays off.

Classification and existence results for $q^{r}$-divisible codes can also be used to decrease upper bounds in the context of subspace codes with mixed dimensions. A concrete numerical example is the following: 
Lemma 11. $A_{3}(9,5) \leqslant 123048$

Proof. Let $\mathcal{C}$ be a subspace code of $\mathbb{F}_{3}^{9}$ with minimum subspace distance $d=5$, and let $a_{i}$ denote the number of $i$-dimensional codewords. W.l.o.g. we assume $a_{0}+a_{1}+a_{2}+a_{3}+a_{4} \geqslant$ $a_{5}+a_{6}+a_{7}+a_{8}+a_{9}$, so that $\# \mathcal{C} \leqslant 2 \sum_{i=0}^{4} a_{i}$. If $a_{0}+a_{1} \geqslant 1$, then $a_{0}+a_{1}=1$ and $\# \mathcal{C} \leqslant 2+2 a_{4} \leqslant 2+2 A_{3}(9,6 ; 4) \leqslant 122022$. In the following we assume $a_{0}=a_{1}=0$.

Next we consider the possible maximal patterns of codewords through a point, i.e., in $\mathbb{F}_{q}^{8}$ we consider sets of codewords with a dimension in $\{1,2,3\}$ and minimum subspace distance at least 5: $1^{1}$ and $2^{x_{2}} 3^{x_{3}}$, where $x_{2} \leqslant 1$ and $x_{3} \leqslant A_{3}(8,6 ; 3)$. For the latter the tightest known bounds are $244 \leqslant A_{3}(8,6 ; 3) \leqslant 248$. If $A_{3}(8,6 ; 3)=248$ then the corresponding 56 holes have to form a $3^{2}$-divisible projective set for which the unique possibility is the Hill cap, see e.g. [19], which does not contain a line. So, no vector space partition of type $1^{52} 2^{1} 3^{248}$ exists in $\mathbb{F}_{3}^{8}$, which implies $x_{2}+x_{3} \leqslant 248$.

Solving ILP (9) with the patterns $1^{1}, 2^{1} 3^{247}, 3^{248}$ and $a_{0}=a_{1}=0, a_{2} \leqslant 1, a_{3} \leqslant 757$, $a_{4} \leqslant 61010$ gives the unique solution $a_{2}=0, a_{3}=757, a_{4}=60768$ with target value 123050 .

Assume for a moment that $a_{3}=757$. In that case the 757 planes form a spread, i.e., each point is covered exactly once. So each point can be contained in at most 247 solids. Thus, $a_{4} \leqslant\left\{247 \cdot\left[\begin{array}{l}9 \\ 1\end{array}\right]_{3} /\left[\begin{array}{l}4 \\ 1\end{array}\right]_{3}\right\}_{4}=60766$. (We have $a_{4} \leqslant\left\lfloor 247 \cdot\left[\begin{array}{l}9 \\ 1\end{array}\right]_{3} /\left[\begin{array}{l}4 \\ 1\end{array}\right]_{3}\right\rfloor=60768$. If $a_{4}=60768$ then there would be a $3^{3}$-divisible linear code of length 7 , If $a_{4}=60767$ then there would be a $3^{3}$-divisible linear code of length 47 , which both do not exist, see [21].) Thus, $\# \mathcal{C} \leqslant 123048$.

If we add $a_{3} \leqslant 756$ to our ILP formulation we also get a target value of 123048 .

We remark that this is a numerical improvement of the more general Lemma 12 . In the next section we apply the underlying general idea of the Johnson bound, as outlined above, to $A_{q}(v, v-4)$ and $A_{q}(8,3)$.

\section{Analytical results}

Lemma 12. For odd $v \geqslant 7$ we have

$$
\begin{aligned}
A_{q}(v, v-4) \leqslant & \max \left\{2 A_{q}(v, v-3 ; m-1)+2 A_{q}(v, v-3 ; m),\right. \\
& 2+2\left\lfloor\left(\left[\begin{array}{c}
2 m+1 \\
1
\end{array}\right]_{q}-\left[\begin{array}{c}
m-2 \\
1
\end{array}\right]_{q}\right) /\left[\begin{array}{c}
m-1 \\
1
\end{array}\right]_{q}\right\rfloor{ }^{2} \\
& \left.+2\left\lfloor\left(\left[\begin{array}{c}
2 m+1 \\
1
\end{array}\right]_{q}-\left[\begin{array}{c}
m-2 \\
1
\end{array}\right]_{q}\right) \cdot A_{q}(2 m, 2 m-2 ; m-1) /\left[\begin{array}{c}
m \\
1
\end{array}\right]_{q}\right\rfloor\right\},
\end{aligned}
$$

where $m=(v-1) / 2$.

Proof. Let $\mathcal{C}$ be a subspace code of $\mathbb{F}_{q}^{v}$ with minimum subspace distance $d=v-4$, $m=\frac{v-1}{2} \geqslant 3$, and $a_{i}$ denote the number of $i$-dimensional codewords. W.l.o.g. we assume 
$\sum_{i=0}^{m} a_{i} \geqslant \sum_{i=m+1}^{v} a_{i}$, so that $\# \mathcal{C} \leqslant 2 \sum_{i=0}^{m} a_{i}$. Since $d=2 m-3$ we have $\sum_{i=0}^{m-2} a_{i} \leqslant 1$. If there exists an index $0 \leqslant i \leqslant m-3$ with $a_{i}>0$, then $\# \mathcal{C} \leqslant 2+2 A_{q}(v, v-3 ; m)$. If $\sum_{i=0}^{m-2} a_{i}=0$, then $\# \mathcal{C} \leqslant 2 A_{q}(v, v-3 ; m-1)+2 A_{q}(v, v-3 ; m)$, which is at least as large as $2+2 A_{q}(v, v-3 ; m)$. It remains to consider the case $a_{m-2}=1$. Here we consider the possible maximal patterns of codewords through a point, i.e., in $\mathbb{F}_{q}^{2 m}$ we consider sets of codewords with a dimension in $\{m-3, m-2, m-1\}$ and minimum subspace distance at least $2 m-3:(m-3)^{1}$ and $(m-2)^{1}(m-1)^{A_{q}(2 m, 2 m-2 ; m-1)}$. The first pattern is attained $\left[\begin{array}{c}m-2 \\ 1\end{array}\right]_{q}$ times so that

$$
a_{m-1} \leqslant\left\lfloor\left(\left[\begin{array}{c}
2 m+1 \\
1
\end{array}\right]_{q}-\left[\begin{array}{c}
m-2 \\
1
\end{array}\right]_{q}\right) /\left[\begin{array}{c}
m-1 \\
1
\end{array}\right]_{q}\right\rfloor
$$

and

$$
a_{m} \leqslant\left\lfloor\left(\left[\begin{array}{c}
2 m+1 \\
1
\end{array}\right]_{q}-\left[\begin{array}{c}
m-2 \\
1
\end{array}\right]_{q}\right) \cdot A_{q}(2 m, 2 m-2 ; m-1) /\left[\begin{array}{c}
m \\
1
\end{array}\right]_{q}\right\rfloor \text {. }
$$

We remark that $\left.\mid\left(\left[\begin{array}{c}2 m+1 \\ 1\end{array}\right]_{q}-\left[\begin{array}{c}m-2 \\ 1\end{array}\right]_{q}\right) /\left[\begin{array}{c}m-1 \\ 1\end{array}\right]_{q}\right\rfloor$ can be simplified to $q^{5}+q^{3}+q$ for $m=3$, $q^{6}+q^{3}$ for $m=4, q^{7}+q^{3}$ for $m=5$, and $q^{m+2}+q^{3}-1$ for $m \geqslant 6$. The upper bound can be improved if there is no vector space partition of type $1^{\star}(m-2)^{1}(m-1)^{A_{q}(2 m, 2 m-2 ; m-1)}$ of $\mathbb{F}_{q}^{2 m}$. This happens e.g. for $m=3$ and arbitrary $q$. Since $A_{q}(6,4 ; 2)=q^{4}+q^{2}+1$ and $A_{q}(7,4 ; 2)=q^{5}+q^{3}+1$ the upper bound of upper bound of Lemma 12 evaluates to $A_{q}(7,3) \leqslant 2\left(q^{8}+q^{6}+2 q^{5}+q^{4}+2 q^{3}+q^{2}+2\right)$ using the Anticode bound $A_{q}(7,4 ; 3) \leqslant$ $\left[\begin{array}{l}7 \\ 1\end{array}\right]_{q} \cdot\left(q^{2}-q+1\right)$, which is the tightest known bound for these parameters. This can be further improved to:

Lemma 13. $A_{q}(7,3) \leqslant 2\left(q^{8}+q^{6}+2 q^{5}+2 q^{3}+q^{2}-q+2\right)$

Proof. Let $\mathcal{C}$ be a subspace code of $\mathbb{F}_{q}^{7}$ with minimum subspace distance $d=3$, and let $a_{i}$ denote the number of $i$-dimensional codewords. W.l.o.g. we assume $a_{0}+a_{1}+a_{2}+a_{3} \geqslant$ $a_{4}+a_{5}+a_{6}+a_{7}$, so that $\# \mathcal{C} \leqslant 2 \sum_{i=0}^{3} a_{i}$. If $a_{0} \geqslant 1$, then $a_{0}=1$ and $\# \mathcal{C} \leqslant 2+2 a_{3} \leqslant$ $2\left(q^{8}+q^{6}+q^{5}+q^{4}+q^{3}+q^{2}+2\right) \leqslant 2\left(q^{8}+q^{6}+2 q^{5}+2 q^{3}+q^{2}-q+2\right)$. Next we consider the possible maximal patterns of codewords through a point, i.e., in $\mathbb{F}_{q}^{6}$ we consider sets of codewords with a dimension in $\{0,1,2\}$ and minimum subspace distance at least 3 : $0^{1}$ and $1^{x_{1}} 2^{x_{2}}$, where $x_{1} \leqslant 1$ and $x_{2} \leqslant A_{q}(6,4 ; 2)=q^{4}+q^{2}+1$. Since $x_{2}=A_{q}(6,4 ; 2)$ can only be attained in case of a line spread, we have $x_{1}+x_{2} \leqslant q^{4}+q^{2}+1$, which gives the possible maximal patterns $1^{1} 2^{q^{4}+q^{2}}$ and $2^{q^{4}+q^{2}+1}$. We start with the case $a_{1}=0$ and denote the multiplicities of the patterns $1^{1} 2^{q^{4}+q^{2}}$ and $2^{q^{4}+q^{2}+1}$ by $m_{1}$ and $m_{2}$, respectively. With this we have $a_{2} \leqslant\left\lfloor m_{1} /\left[\begin{array}{l}2 \\ 1\end{array}\right]_{q}\right\rfloor$ and $a_{3} \leqslant\left\lfloor\left(\left[\begin{array}{l}7 \\ 1\end{array}\right]_{q} \cdot\left(q^{4}+q^{2}+1\right)-m_{1}\right) /\left[\begin{array}{l}3 \\ 1\end{array}\right]_{q}\right\rfloor$, so that

$$
\begin{aligned}
\# \mathcal{C} & \leqslant 2\left(m_{1} /\left[\begin{array}{l}
2 \\
1
\end{array}\right]_{q}+\left(\left[\begin{array}{l}
7 \\
1
\end{array}\right]_{q} \cdot\left(q^{4}+q^{2}+1\right)-m_{1}\right) /\left[\begin{array}{l}
3 \\
1
\end{array}\right]_{q}\right) \\
& =2 m_{1} \cdot \frac{q^{2}}{(q+1)\left(q^{2}+q+1\right)}+2\left[\begin{array}{l}
7 \\
1
\end{array}\right]_{q} \cdot\left(q^{2}-q+1\right)=: f\left(m_{1}\right),
\end{aligned}
$$


which is increasing in $m_{1}$. Next we invoke $\# \mathcal{C} \leqslant 2 a_{2}+2 a_{3}$ and $a_{2} \leqslant A_{q}(7,4 ; 2)=q^{5}+q^{3}+1$. If $m_{1} \geqslant\left[\begin{array}{l}2 \\ 1\end{array}\right]_{q} \cdot\left(q^{5}+q^{3}+1\right)$, then $a_{2} \leqslant q^{5}+q^{3}+1$ and

$$
\begin{aligned}
a_{3} & \leqslant\left\lfloor\left(\left[\begin{array}{l}
7 \\
1
\end{array}\right]_{q} \cdot\left(q^{4}+q^{2}+1\right)-\left[\begin{array}{l}
2 \\
1
\end{array}\right]_{q} \cdot\left(q^{5}+q^{3}+1\right)\right) /\left[\begin{array}{l}
3 \\
1
\end{array}\right]_{q}\right\rfloor \\
& =\left[\begin{array}{l}
7 \\
1
\end{array}\right]_{q} \cdot\left(q^{2}-q+1\right)-\left\lceil\frac{(q+1) \cdot\left(q^{5}+q^{3}+1\right)}{q^{2}+q+1}\right\rceil \\
& =\left[\begin{array}{l}
7 \\
1
\end{array}\right]_{q} \cdot\left(q^{2}-q+1\right)-q^{4}-q+\left\lfloor\frac{q^{2}-1}{q^{2}+q+1}\right\rfloor \\
& =q^{8}+q^{6}+q^{5}+q^{3}+q^{2}-q+1,
\end{aligned}
$$

so that $\# \mathcal{C} \leqslant 2\left(q^{8}+q^{6}+2 q^{5}+2 q^{3}+q^{2}-q+2\right)$. If $\left[\begin{array}{l}2 \\ 1\end{array}\right]_{q} \cdot\left(q^{5}+q^{3}\right) \leqslant m_{1}<\left[\begin{array}{l}2 \\ 1\end{array}\right]_{q} \cdot\left(q^{5}+q^{3}+1\right)$, then $a_{2} \leqslant A_{2}(7,4 ; 2)-1=q^{5}+q^{3}$ so that

$$
\# \mathcal{C} \leqslant f\left((q+1) \cdot\left(q^{5}+q^{3}\right)\right)=2\left(q^{8}+q^{6}+2 q^{5}+2 q^{3}+q^{2}-q+2-\frac{1}{q^{2}+q+1}\right) .
$$

If $a_{1}=1$, then

$$
\begin{aligned}
\# \mathcal{C} & \leqslant 2\left(1+m_{1} /\left[\begin{array}{l}
2 \\
1
\end{array}\right]_{q}+\left(\left[\begin{array}{l}
7 \\
1
\end{array}\right]_{q} \cdot\left(q^{4}+q^{2}+1\right)-m_{1}-\left(q^{4}+q^{2}+1\right)\right) /\left[\begin{array}{l}
3 \\
1
\end{array}\right]_{q}\right) \\
& =2\left(m_{1} \cdot \frac{q^{2}}{(q+1)\left(q^{2}+q+1\right)}+\left[\begin{array}{l}
7 \\
1
\end{array}\right]_{q} \cdot\left(q^{2}-q+1\right)-\left(q^{2}-q\right)\right) .
\end{aligned}
$$

Since we can assume $m_{1} \leqslant(q+1)\left(q^{5}+q^{3}+1\right)$ we have $\# \mathcal{C} \leqslant 2\left(q^{8}+q^{6}+2 q^{5}+2 q^{3}+3\right)$.

In the binary case Lemma 13 gives the upper bound $A_{2}(7,3) \leqslant 808$ while the semidefinite programming method from [2] gives $A_{2}(7,3) \leqslant 776$. Also for $3 \leqslant q \leqslant 7$ the semidefinite programming method gives tighter upper bounds, see [10].

Lemma 14. Let $m \geqslant 4$. If $A_{q}(2 m, 2 m-4)>2+A_{q}(2 m, 2 m-4 ; m)$, then we have

$$
A_{q}(2 m, 2 m-4) \leqslant\left\lfloor\frac{\left[\begin{array}{c}
2 m \\
1
\end{array}\right]_{q}}{\left[\begin{array}{c}
m \\
1
\end{array}\right]_{q}} \cdot\left\lfloor\frac{\left(\left[\begin{array}{c}
2 m-1 \\
1
\end{array}\right]_{q}-\left[\begin{array}{c}
m-3 \\
1
\end{array}\right]_{q}\right) \cdot A_{q}(2 m-2,2 m-4 ; m-2)}{\left[\begin{array}{c}
m-1 \\
1
\end{array}\right]_{q}}\right\rfloor+\frac{2\left[\begin{array}{c}
2 m \\
1
\end{array}\right]_{q}}{\left[\begin{array}{c}
m-2 \\
1
\end{array}\right]_{q}}\right\rfloor
$$

if $m=4$ or $(m=5$ and $q=2)$ and

$$
A_{q}(2 m, 2 m-4) \leqslant\left\lfloor\frac{\left[\begin{array}{c}
2 m \\
1
\end{array}\right]_{q}}{\left[\begin{array}{c}
m \\
1
\end{array}\right]_{q}} \cdot\left\lfloor\frac{\left[\begin{array}{c}
2 m-1 \\
1
\end{array}\right]_{q} \cdot A_{q}(2 m-2,2 m-4 ; m-2)}{\left[\begin{array}{c}
m-1 \\
1
\end{array}\right]_{q}}\right\rfloor\right\rfloor
$$

otherwise. 
Proof. Let $\mathcal{C}$ be a subspace code of $\mathbb{F}_{2}^{2 m}$ with minimum subspace distance $d=2 m-4$, and let $a_{i}$ denote the number of $i$-dimensional codewords, so that $\# \mathcal{C}=\sum_{i=0}^{2 m} a_{i}$. Due to duality we assume $\sum_{i=0}^{m-1} a_{i} \geqslant \sum_{i=m+1}^{2 m} a_{i}$, so that $\# \mathcal{C} \leqslant a_{m}+2 \sum_{i=0}^{m-1} a_{i}$. If $a_{i} \geqslant 1$ for an index $0 \leqslant i \leqslant m-4$, then $\# \mathcal{C} \leqslant 2+A_{q}(2 m, 2 m-4)$, so that we assume $a_{i}=0$ for all $0 \leqslant i \leqslant m-4$ in the following. Next we consider the possible maximal patterns of codewords through a point, i.e., in $\mathbb{F}_{q}^{2 m-1}$ we consider sets of codewords with a dimension in $\{m-4, m-3, m-2, m-1\}$ and minimum subspace distance at least $2 m-4:(m-4)^{1}$, $(m-3)^{1}(m-1)^{x}$, and $(m-2)^{a}(m-1)^{b}$, where we have to determine the possible values for $x, a$, and $b$. To that end we consider the possible maximal patterns of codewords in $\mathbb{F}_{q}^{2 m-2}$ with a dimension in $\{m-4, m-3, m-2\}$ and minimum subspace distance at least $2 m-4:(m-4)^{1},(m-3)^{1}$, and $(m-2)^{\tau}$, where $\tau:=A_{q}(2 m-2,2 m-4 ; m-2)$. Thus, we can choose

$$
x=\left\lfloor\frac{\left.\left(\begin{array}{c}
2 m-1 \\
1
\end{array}\right]_{q}-\left[\begin{array}{c}
m-3 \\
1
\end{array}\right]_{q}\right) \cdot \tau}{\left[\begin{array}{c}
m-1 \\
1
\end{array}\right]_{q}}\right\rfloor
$$

and have

$$
b \leqslant\left\lfloor\frac{\left.\left(\begin{array}{c}
2 m-1 \\
1
\end{array}\right]_{q}-a\left[\begin{array}{c}
m-2 \\
1
\end{array}\right]_{q}\right) \cdot \tau}{\left[\begin{array}{c}
m-1 \\
1
\end{array}\right]_{q}}\right\rfloor,
$$

where $a \in \mathbb{N}$. Since $\left[\begin{array}{c}m-2 \\ 1\end{array}\right]_{q} \geqslant 2$ and $\tau=A_{q}(2 m-2,2 m-4 ; m-2) \geqslant q^{m} \geqslant\left[\begin{array}{c}m \\ 1\end{array}\right]_{q}$ we have

$$
\frac{2 a}{\left[\begin{array}{c}
m-1 \\
1
\end{array}\right]_{q}}-\frac{\tau\left[\begin{array}{c}
m-2 \\
1
\end{array}\right]_{q} \cdot a}{\left[\begin{array}{c}
m-1 \\
1
\end{array}\right]_{q} \cdot\left[\begin{array}{c}
m \\
1
\end{array}\right]_{q}} \leqslant 0
$$

so that the score for pattern $(m-2)^{a}(m-1)^{b}$ is decreasing in $a$. For $a=0$ we obtain a score of

$$
s_{3}:=\frac{1}{\left[\begin{array}{c}
m \\
1
\end{array}\right]_{q}} \cdot\left\lfloor\frac{\left[\begin{array}{c}
2 m-1 \\
1
\end{array}\right]_{q} \cdot \tau}{\left[\begin{array}{c}
m-1 \\
1
\end{array}\right]_{q}}\right\rfloor .
$$

For pattern $(m-3)^{1}(m-1)^{x}$ the score is given by

$$
s_{2}:=\frac{1}{\left[\begin{array}{c}
m \\
1
\end{array}\right]_{q}} \cdot\left\lfloor\frac{\left.\left(\begin{array}{c}
2 m-1 \\
1
\end{array}\right]_{q}-\left[\begin{array}{c}
m-3 \\
1
\end{array}\right]_{q}\right) \cdot \tau}{\left[\begin{array}{c}
m-1 \\
1
\end{array}\right]_{q}}\right\rfloor+\frac{2}{\left[\begin{array}{c}
m-2 \\
1
\end{array}\right]_{q}}
$$

and for $(m-4)^{1}$ we have a score of $s_{1}:=\frac{2}{\left[\begin{array}{c}m-3 \\ 1\end{array}\right]_{q}}$. In order to compare the three scores we use $q^{m}+1 \leqslant \tau=A_{q}(2 m-2,2 m-4 ; m-2) \leqslant\left[\begin{array}{c}2 m-2 \\ 1\end{array}\right]_{q} /\left[\begin{array}{c}m-2 \\ 1\end{array}\right]_{q}$ and $q^{k-1}+1 \leqslant\left[\begin{array}{l}k \\ 1\end{array}\right]_{q} \leqslant 2 q^{k-1}-1$ for $k \geqslant 2$. Obviously, we have $s_{3} \geqslant s_{1}$. If $m=4$, then using $\left[\begin{array}{l}1 \\ 1\end{array}\right]_{q}=1$ gives

$$
\begin{aligned}
s_{2}-s_{3} & \geqslant-\frac{1}{\left[\begin{array}{c}
m \\
1
\end{array}\right]_{q}} \cdot \frac{\left[\begin{array}{c}
m-3 \\
1
\end{array}\right]_{q} \cdot \tau+1}{\left[\begin{array}{c}
m-1 \\
1
\end{array}\right]_{q}}+\frac{2}{\left[\begin{array}{c}
m-2 \\
1
\end{array}\right]_{q}} \\
& \geqslant \frac{1}{\left[\begin{array}{c}
m-2 \\
1
\end{array}\right]_{q}} \cdot\left(2-\frac{2 q^{2 m-3}}{q^{m-1} q^{m-2}}\right) \geqslant 0 .
\end{aligned}
$$


In the other direction we have

$$
\begin{aligned}
s_{2}-s_{3} & \leqslant-\frac{1}{\left[\begin{array}{c}
m \\
1
\end{array}\right]_{q}} \cdot \frac{\left[\begin{array}{c}
m-3 \\
1
\end{array}\right]_{q} \cdot \tau-1}{\left[\begin{array}{c}
m-1 \\
1
\end{array}\right]_{q}}+\frac{2}{\left[\begin{array}{c}
m-2 \\
1
\end{array}\right]_{q}} \\
& \leqslant \frac{2}{q^{m-3}}-\frac{1}{2 q^{m-1}} \cdot\left(\frac{q^{m-4} \cdot q^{m}}{2 q^{m-2}}-1\right) \\
& \leqslant \frac{2}{q^{m-3}} \cdot\left(1-\frac{1}{4 q^{2}} \cdot\left(\frac{q^{m-2}}{2}-1\right)\right),
\end{aligned}
$$

which is negative if $m \geqslant 7$ or $m=6$ and $q \geqslant 3$. For $m=6$ and $q=2$ we plug in the known numerical values for the first inequality and obtain $s_{2}-s_{3}<0$. It remains to consider $m=5$, where $A_{q}(8,6,3) \geqslant q^{5}+1$ and

$$
\begin{aligned}
s_{2}-s_{3} & \leqslant-\frac{1}{\left[\begin{array}{l}
5 \\
1
\end{array}\right]_{q}} \cdot\left\lfloor\frac{(q+1) \tau-1}{q^{3}+q^{2}+q+1}\right\rfloor+\frac{2}{q^{2}+q+1} \\
& \leqslant-\frac{q^{3}-q}{q^{4}+q^{3}+q^{2}+q+1}+\frac{2}{q^{2}+q+1},
\end{aligned}
$$

which is negative for $q \geqslant 3$. For $m=5$ and $q=2$ we can easily check have $s_{2}-s_{3}>0$.

The next lemma shows that a specific configuration consisting of a point, some lines and some planes does not exist in $\mathbb{F}_{q}^{7}$. This result will then be used to proof an upper bound on $A_{q}(8,3)$.

Lemma 15. There exists no subspace code $\mathcal{C}$ in $\mathbb{F}_{q}^{7}$ with minimum subspace distance $d=3$ and dimension distribution $1^{1} 2^{q^{4}+q^{2}+2} 3^{q^{8}+q^{6}+q^{5}+q^{3}}$.

Proof. Assume that $\mathcal{C}$ is a code in $V=\mathbb{F}_{q}^{7}$ of minimum subspace distance 3 containing a single point $P$ and $q^{8}+q^{6}+q^{5}+q^{3}$ planes. We denote the set of lines in $\mathcal{C}$ by $\mathcal{C}_{2}$ and the set of planes in $\mathcal{C}$ by $\mathcal{C}_{3}$. As the subspace distance is at least $3, P$ is not contained in any element of $\mathcal{C}_{2}$ and $\mathcal{C}_{3}$, no line in $\mathcal{C}_{2}$ is contained in a plane in $\mathcal{C}_{3}$, the lines in $\mathcal{C}_{2}$ are pairwise disjoint and the pairwise intersection of the planes in $\mathcal{C}_{3}$ is at most a point. The lines in the ambient space not covered by any plane in $\mathcal{C}_{3}$ will be called free. All lines in $\mathcal{C}_{2}$ and all lines passing through $P$ are free.

For a point $Q$, let $\mathcal{C}_{3}(Q)$ be the set of all planes in $\mathcal{C}_{3}$ passing through $Q$. Clearly, $\mathcal{C}_{3}(P)=\emptyset$. For $Q \neq P, \# \mathcal{C}(Q) \leqslant q^{4}+q^{2}$, since otherwise all the points of the ambient space, including $P$, would be covered by some element in $\mathcal{C}_{3}(Q) .{ }^{1}$ We count the set $X$ of flags $(Q, E)$ with $Q \in\left[\begin{array}{l}V \\ 1\end{array}\right]$ and $E \in \mathcal{C}_{3}$ in two ways. Since $Q<E$,

$$
\# X=\# \mathcal{C}_{3} \cdot\left[\begin{array}{l}
3 \\
1
\end{array}\right]_{q}=q^{3}\left(q^{2}+1\right)\left(q^{3}+1\right) \cdot\left(q^{2}+q+1\right) .
$$

\footnotetext{
${ }^{1}$ For $\# \mathcal{C}_{3}(Q)=q^{4}+q^{2}+1$, the image of $\mathcal{C}_{3}(Q)$ modulo $Q$ would be a line spread in $V / Q \cong \mathbb{F}_{q}^{6}$.
} 
On the other hand,

$$
\# X=\sum_{Q \in\left[\begin{array}{l}
V \\
1
\end{array}\right]} \# \mathcal{C}_{3}(Q) \leqslant\left(\left[\begin{array}{l}
7 \\
1
\end{array}\right]_{q}-1\right) \cdot\left(q^{4}+q^{2}\right)=q\left(q^{3}+1\right)\left(q^{2}+q+1\right) \cdot q^{2}\left(q^{2}+1\right) .
$$

Thus, we have in fact equality, which implies $\# \mathcal{C}_{3}(Q)=q^{4}+q^{2}$ for all $Q \neq P$.

Modulo $Q$, the $q^{4}+q^{2}$ planes in $\mathcal{C}_{3}(Q)$ form a partial line spread in $V / Q$. It is known that every such partial spread is extendible to a spread. ${ }^{2}$ Therefore, the set of $q+1$ free lines through $Q$ spans a plane $E(Q)$, and all lines in $E(Q)$ passing through $Q$ are free.

Let $Q^{\prime} \in E(Q) \backslash\{P\}$. For $Q^{\prime} \notin\langle P, Q\rangle, E(Q)$ contains the distinct free lines $\left\langle Q^{\prime}, P\right\rangle$ and $\left\langle Q^{\prime}, Q\right\rangle$, implying that $E\left(Q^{\prime}\right)=E(Q)$. For $Q^{\prime} \in\langle P, Q\rangle$, we pick an auxiliary point $Q^{\prime \prime} \in E(Q)$ with $Q^{\prime \prime} \notin\langle P, Q\rangle=\left\langle P, Q^{\prime}\right\rangle$. By applying the previous case twice we get again that $E\left(Q^{\prime}\right)=E\left(Q^{\prime \prime}\right)=E(Q)$. Thus, the set $S=\left\{E(Q) \mid Q \in\left[\begin{array}{l}V \\ 1\end{array}\right] \backslash\{P\}\right\}$ is of size

$$
\frac{\left[\begin{array}{l}
7 \\
1
\end{array}\right]_{q}-1}{\left[\begin{array}{l}
3 \\
1
\end{array}\right]_{q}-1}=\frac{q^{7}-q}{q^{3}-q}=q^{4}+q^{2}+1 .^{3}
$$

Every line $L \in \mathcal{C}_{2}$ is free and therefore contained in a plane $E(Q)$ (any point $Q$ on $L$ does the job). Moreover, a plane $E(Q)$ cannot contain more than one line from $\mathcal{C}_{2}$, as any two lines in a plane intersect. Therefore $\# \mathcal{C}_{2} \leqslant \# S=q^{4}+q^{2}+1$.

Remark 16. The proof of Lemma 15 shows that the free lines are precisely those contained in the planes $E(Q)$. Thus, $\mathcal{C}_{3} \cup S$ covers each line in $\mathrm{PG}(V)$ exactly once. Such a set of planes is known as a $q$-analog of the Fano plane. The question for its existence is open for every single value of $q$ and arguably the most important open problem in the theory of $q$-analogs of designs, see [4] for a survey.

Proposition 17. $A_{q}(8,3) \leqslant q^{12}+3 q^{10}+q^{9}+3 q^{8}+3 q^{7}+3 q^{6}+5 q^{5}+3 q^{4}+q^{3}+4 q^{2}+2 q-1$ for $q \geqslant 3$ and $A_{2}(8,3) \leqslant 9260$.

Proof. Let $\mathcal{C}$ be a subspace code of $\mathbb{F}_{q}^{8}$ with minimum subspace distance $d=3$ and $a_{i}$ denote the number of its codewords of dimension $i$. Due to duality we can assume $\# \mathcal{C} \leqslant 2 \cdot\left(a_{0}+a_{1}+a_{2}+a_{3}\right)+a_{4}$. Of course we have $a_{i} \leqslant A_{q}(8,4 ; i)$ for all $0 \leqslant i \leqslant 4$, i.e., $a_{0}, a_{1} \leqslant 1, a_{2} \leqslant q^{6}+q^{4}+q^{2}+1$,

$$
\begin{aligned}
a_{4} & \leqslant\left[\begin{array}{l}
8 \\
3
\end{array}\right]_{q} /\left[\begin{array}{l}
4 \\
3
\end{array}\right]_{q}=\left(q^{2}-q+1\right)\left(q^{4}+1\right)\left[\begin{array}{l}
7 \\
1
\end{array}\right]_{q} \\
& =q^{12}+q^{10}+q^{9}+2 q^{8}+q^{7}+2 q^{6}+q^{5}+2 q^{4}+q^{3}+q^{2}+1,
\end{aligned}
$$

and

$$
\begin{aligned}
a_{3} & \leqslant\left\lfloor\frac{A_{q}(7,4 ; 2) \cdot\left[\begin{array}{l}
8 \\
1
\end{array}\right]_{q}}{\left[\begin{array}{l}
3 \\
1
\end{array}\right]_{q}}\right\rfloor=\left\lfloor\frac{\left(q^{5}+q^{3}+1\right) \cdot\left(q^{8}-1\right)}{q^{3}-1}\right\rfloor \\
& =q^{10}+q^{8}+q^{7}+2 q^{5}+q^{4}+q^{2}+q-1,
\end{aligned}
$$

\footnotetext{
${ }^{2}$ For example, using that its set of $q+1$ uncovered points corresponds to a linear code of (effective) length $q+1$ whose codewords have a weight that is divisible by $q$. Thus, all non-zero codewords have a weight of $q$ and the corresponding point set has to be a line.

${ }^{3}$ The image of $S$ modulo $P$ is a line spread in $V / P \cong \mathbb{F}_{q}^{6}$.
} 
since $\left\lfloor\frac{q+2}{q^{2}+q+1}\right\rfloor=0$.

If $a_{0}=1$, then $a_{1}=a_{2}=0$, so that

$$
\# \mathcal{C} \leqslant q^{12}+3 q^{10}+q^{9}+4 q^{8}+3 q^{7}+2 q^{6}+5 q^{5}+4 q^{4}+q^{3}+3 q^{2}+2 q+1 .
$$

Thus, we can assume $a_{0}=0$ in the following and consider the set of codewords containing a point $P$. Modulo $P$ the dimension distribution is given by $0^{b_{0}} 1^{b_{1}} 2^{b_{2}} 3^{b_{3}}$, where obviously $b_{i} \leqslant A_{q}(7,4 ; i)$ for $0 \leqslant i \leqslant 3$, i.e., $b_{0}, b_{1} \leqslant 1, b_{2} \leqslant q^{5}+q^{3}+1$, and $b_{3} \leqslant\left[\begin{array}{l}7 \\ 2\end{array}\right]_{q} /\left[\begin{array}{l}3 \\ 2\end{array}\right]_{q}=$ $\left[\begin{array}{l}7 \\ 1\end{array}\right]_{q} \cdot\left(q^{2}-q+1\right)=q^{8}+q^{6}+q^{5}+q^{4}+q^{3}+q^{2}+1$. To each possible dimension distribution we assign a score

$$
\Gamma_{8}(b)=\frac{b_{3}}{\left[\begin{array}{l}
4 \\
1
\end{array}\right]_{q}}+2 \cdot \sum_{i=0}^{2} \frac{b_{i}}{\left[\begin{array}{c}
i+1 \\
1
\end{array}\right]_{q}} .
$$

If the score of each dimension distribution that occurs at a point $P$ in $\mathcal{C}$ is upper bounded by $\omega$, then we have $\# \mathcal{C} \leqslant \omega \cdot\left[\begin{array}{l}8 \\ 1\end{array}\right]_{q}$. The score of a vector $b \in \mathbb{N}^{4}$ is of course at least as large as the score of a vector $b^{\prime} \in \mathbb{N}^{4}$ if $b \geqslant b^{\prime}$ componentwise, so that we just have to consider the feasible dimension distributions that are maximal with respect to this relation. These are given by

(1) $0^{1} 3^{b_{3}}$, where $b_{3}=\left[\begin{array}{l}7 \\ 1\end{array}\right]_{q} \cdot\left(q^{2}-q+1\right)$;

(2) $1^{1} 2^{b_{2}} 3^{b_{3}}$, where $0 \leqslant b_{2} \leqslant q^{5}+q^{3}+1$, and $b_{3} \leqslant \frac{\left(\left[\begin{array}{l}7 \\ 1\end{array}\right]_{q}-1\right) \cdot\left(q^{4}+q^{2}\right)}{q^{2}+q+1}$;

(3) $2^{b_{2}} 3^{b_{3}}$, where $0 \leqslant b_{2} \leqslant q^{5}+q^{3}+1$, and $b_{3} \leqslant \frac{\left[\begin{array}{l}7 \\ 1\end{array}\right]_{q}\left(q^{4}+q^{2}+1\right)-(q+1) b_{2}}{q^{2}+q+1}$.

If $b_{0}=1$, then $b_{1}=b_{2}=0$, which gives case (1). For the remaining cases we may again consider the set of codewords modulo a common point, which then live in $\mathbb{F}_{q}^{6}$. Here the possible maximal dimension distributions are

- $0^{1}$

- $1^{1} 2^{q^{4}+q^{2}}$;

- $2^{q^{4}+q^{2}+1}$.

If $b_{1}=0$ we can directly conclude the stated upper bound for $b_{3}$ in case (3). If $b_{1}=1$ we observe that no $q^{4}+q^{2}+1$ planes can meet in a common point, cf. the proof of Lemma 15, so that we obtain the stated upper bound for $b_{3}$ in case (2). Of course we may round down the, eventually fractional, upper bound for $b_{3}$ to an integer. The scores for the three unrounded cases are given by

(1) $q^{5}-q^{4}+q^{3}+q+1+\frac{q^{2}+2}{q^{3}+q^{2}+q+1}$;

(2) $\frac{2}{q+1}+\left(q^{2}-q+1\right) q^{3}+\frac{2 b_{2}}{q^{2}+q+1}$; 
(3) $\frac{\left[\begin{array}{l}7 \\ 1\end{array}\right]_{q}\left(q^{2}-q+1\right)}{(q+1)\left(q^{2}+1\right)}+\frac{2 q^{2}+1}{\left(q^{2}+1\right)\left(q^{2}+q+1\right)} \cdot b_{2}$.

In cases (2) and (3) the scores are strictly increasing in $b_{2}$ (which also remains valid if be round the upper bound for $b_{3}$ to an integer). Plugging in $b_{2}=q^{5}+q^{3}+1$ gives the following upper bounds for the scores

(1) $q^{5}-q^{4}+q^{3}+q+1+\frac{q^{2}+2}{q^{3}+q^{2}+q+1}$;

(2) $q^{5}-q^{4}+3 q^{3}-2 q^{2}+2 q+\frac{2 q+4}{q^{3}+2 q^{2}+2 q+1}$;

(3) $q^{5}-q^{4}+3 q^{3}-2 q^{2}+2 q+\frac{-q^{4}+2 q^{2}+2}{q^{5}+2 q^{4}+3 q^{3}+3 q^{2}+2 q+1}$.

So, case (2) gives the largest upper bound for the score for all $q$ so that we would obtain an upper bound for $A_{q}(8,3)$. For $q=2$ we would obtain $A_{2}(8,3) \leqslant\lfloor 9277.142857\rfloor=9277$. However, this bound can be slightly improved. The stated score for case (2) corresponds to a subspace code in $\mathbb{F}_{q}^{7}$ with dimension distribution $1^{1} 2^{q^{5}+q^{3}+1} 3^{q^{8}+q^{6}+q^{3}}$, i.e., the code excluded in Lemma 15. We can easily check that the second best score in case (2) is obtained if a plane is removed. In case (3) we can perform the rounding for the upper bound for $b_{3}$, which gives $b_{3} \leqslant q^{8}+q^{6}+q^{5}+q^{3}+q^{2}-q+1$ for $b_{2}=q^{5}+q^{3}+1$, since $\left\lfloor\frac{-q-2}{q^{2}+q+1}\right\rfloor=-1$. (Decreasing $b_{2}$ instead gives a lower score, even without rounding the corresponding upper bound for $b_{3}$.) Thus, we obtain the following improved upper bounds for the scores

(1) $0^{1} 3^{q^{8}+q^{6}+q^{5}+q^{4}+q^{3}+q^{2}+1}: q^{5}-q^{4}+q^{3}+q+1+\frac{q^{2}+2}{q^{3}+q^{2}+q+1}$;

(2)

$$
\begin{aligned}
& \text { (2) } 1^{1} 2^{q^{5}+q^{3}+1} 3^{q^{8}+q^{6}+q^{5}+q^{3}-1}: q^{5}-q^{4}+3 q^{3}-2 q^{2}+2 q+\overbrace{\frac{2 q^{3}+3 q^{2}+q+3}{q^{5}+2 q^{4}+3 q^{3}+3 q^{2}+2 q+1}}^{<1} \text {; } \\
& \text { (3) } 2^{q^{5}+q^{3}+1} 3^{q^{8}+q^{6}+q^{5}+q^{3}+q^{2}-q+1}: q^{5}-q^{4}+3 q^{3}-2 q^{2}+2 q+\frac{-q^{4}+q^{2}+3}{q^{5}+2 q^{4}+3 q^{3}+3 q^{2}+2 q+1}
\end{aligned}
$$

Again case (2) obtains the best score, then case (3), and then case (1). For $q=2$, we obtain $A_{2}(8,3) \leqslant\lfloor 9260.142856\rfloor=9260$. Since $\left\lfloor\frac{2 q+4}{q^{2}+q+1}\right\rfloor=0$ for $q \geqslant 3$, we obtain an upper bound of

$$
A_{q}(8,3) \leqslant q^{12}+3 q^{10}+q^{9}+3 q^{8}+3 q^{7}+3 q^{6}+5 q^{5}+3 q^{4}+q^{3}+4 q^{2}+2 q-1 .
$$

We remark that the upper bound of Proposition 17 can almost surely be decreased by 1 , since the considered fractional solution violates $a_{3} \leqslant A_{q}(8,4 ; 3)$ by $1-\frac{3}{q^{2}+q+1}$. However, the corresponding analysis might get quite involved, i.e., one has to solve an ILP.

Given the proof of Lemma 15, it seems more reasonable to also exclude cases where the number of planes is strictly less than $q^{8}+q^{6}+q^{5}+q^{3}$. For $q=2$ the later number is 360 while the largest known number of planes in $\mathbb{F}_{2}^{7}$ with subspace distance $d=3$ is 333 [11]. We remark that the exclusion of dimension distribution $1^{1} 2^{q^{5}+q^{3}} 3^{q^{8}+q^{6}+q^{5}+q^{3}}$ in $\mathbb{F}_{q}^{7}$ for subspace distance $d=3$ would have been sufficient for the conclusion in the proof of Proposition 17. However, we think that Lemma 15 is interesting in its own right and the presented tightening does not complicate the proof. 


\section{Conclusion}

We have generalized the underlying idea of the Johnson bound for constant dimension codes to mixed dimension subspace codes. As in the case of the Etzion-Vardy ILP we also have to deal with integer linear programs. However, things get more complicated. Nevertheless parametric improved upper bounds for $A_{q}(v, v-4)$ and $A_{q}(8,3)$ have been obtained. We illustrate our results with a small table of improvements for the binary case and small parameters:

\begin{tabular}{lllllll}
\hline parameters & improved cdc & ILP E/V & SDP & johnson & details & bklb \\
\hline$A_{2}(10,5)$ & 48394 & 48336 & 49394 & 48104 & Lemma 10 & 32940 \\
$A_{2}(10,6)$ & 48394 & 48336 & - & 38275 & Lemma 14 & 32890 \\
$A_{2}(11,7)$ & 8844 & $9120^{\star}$ & 8990 & 8842 & Lemma 12 & 8067 \\
$A_{2}(13,9)$ & 34058 & $34591^{\star}$ & 34306 & 34056 & Lemma 12 & 32514 \\
\hline
\end{tabular}

Here "improved cdc" refers to Lemma 1, "ILP E/V" to the ILP of Etzion and Vardy, see Section 2, "SDP" to results based on semidefinite programming, see [2], "johnson" to the results obtained in this paper, and "bklb" to the currently best known lower bound, see [12]. If the entry of "ILP E/V" is written in italics, then the value for subspace distance $d-1$ is taken. If the entry is marked with ${ }^{\star}$ then the value of [2] is taken.

\section{Acknowledgement}

The authors are grateful to one reviewer for valuable comments and corrections.

\section{References}

[1] C. Bachoc, D. C. Gijswijt, A. Schrijver, and F. Vallentin. Invariant semidefinite programs. In Handbook on semidefinite, conic and polynomial optimization, pages 219-269. Springer, 2012.

[2] C. Bachoc, A. Passuello, and F. Vallentin. Bounds for projective codes from semidefinite programming. Advances in Mathematics of Communications, 7(2):127-145, 2013.

[3] A. Beutelspacher. Partial spreads in finite projective spaces and partial designs. Mathematische Zeitschrift, 145(3):211-229, 1975.

[4] M. Braun, M. Kiermaier, and A. Wassermann q-analogs of designs: Subspace designs. In Network Coding and Subspace Designs (M. Greferath, M. O. Pavčević, N. Silberstein, and M. Á. Vázquez-Castro, eds.), Signals and Communication Theory, Springer, Cham, 2018, pages 171-211.

[5] D. Drake and J. Freeman. Partial $t$-spreads and group constructible $(s, r, \mu)$-nets. Journal of Geometry, 13(2):210-216, 1979. 
[6] T. Etzion and L. Storme. Galois geometries and coding theory. Designs, Codes and Cryptography, 78(1):311-350, 2016.

[7] T. Etzion and A. Vardy. Error-correcting codes in projective space. IEEE Transactions on Information Theory, 57(2):1165-1173, 2011.

[8] M. Greferath, M. Pavčević, N. Silberstein, and M. Á. Vàzquez-Castro, editors. Network Coding and Subspace Designs. Springer, 2017.

[9] D. Heinlein, T. Honold, M. Kiermaier, S. Kurz, and A. Wassermann. Classification of optimal binary subspace codes of length 8, constant dimension 4 and minimum distance 6. Designs, Codes and Cryptography, 87(2-3):375-391, 2019.

[10] D. Heinlein and F. Ihringer- New and updated semidefinite programming bounds for subspace codes. arXiv:1809.09352, 2018.

[11] D. Heinlein, M. Kiermaier, S. Kurz, and A. Wassermann. A subspace code of size 333 in the setting of a binary $q$-analog of the Fano plane. Advances in Mathematics of Communications, 13(3):457-475, 2019.

[12] D. Heinlein, M. Kiermaier, S. Kurz, and A. Wassermann. Tables of subspace codes. arXiv: 1601.02864, 2016.

[13] D. Heinlein and S. Kurz. Asymptotic bounds for the sizes of constant dimension codes and an improved lower bound. In 5th International Castle Meeting on Coding Theory and Applications, pages 1-30, 2017. arXiv:1705.03835.

[14] D. Heinlein and S. Kurz. An upper bound for binary subspace codes of length 8, constant dimension 4 and minimum distance 6. In The Tenth International Workshop on Coding and Cryptography, pages 1-9, 2017. arXiv preprint 1703.08712.

[15] D. Heinlein and S. Kurz. Binary subspace codes in small ambient spaces. Advances in Mathematics of Communications, 13(4):817-839, 2018.

[16] T. Honold, M. Kiermaier, and S. Kurz. Optimal binary subspace codes of length 6, constant dimension 3 and minimum subspace distance 4. In Topics in finite fields, volume 632 of Contemp. Math., pages 157-176. Amer. Math. Soc., Providence, RI, 2015.

[17] T. Honold, M. Kiermaier, and S. Kurz. Classification of large partial plane spreads in $P G(6,2)$ and related combinatorial objects. Journal of Geometry, 110(5):1-31, 2019.

[18] T. Honold, M. Kiermaier, and S. Kurz. Constructions and bounds for mixeddimension subspace codes. Advances in Mathematics of Communication, 10(3):649$682,2016$.

[19] T. Honold, M. Kiermaier, and S. Kurz. Partial spreads and vector space partitions. In Network Coding and Subspace Designs (M. Greferath, M. O. Pavčević, N. Silberstein, and M. Á. Vázquez-Castro, eds.), Signals and Communication Theory, Springer, Cham, 2018, pages 131-170.

[20] S. Johnson. A new upper bound for error-correcting codes. IRE Transactions on Information Theory, 8(3):203-207, 1962. 
[21] M. Kiermaier and S. Kurz. An improvement of the Johnson bound for subspace codes. arXiv:1707.00650, 2017.

[22] S. Kurz. Improved upper bounds for partial spreads. Designs, Codes and Cryptography, 85(1):97-106, 2017.

[23] S. Kurz. Packing vector spaces into vector spaces. The Australasian Journal of Combinatorics, 68(1):122-130, 2017.

[24] F. J. MacWilliams and N. J. A. Sloane. The theory of error-correcting codes. II. North-Holland Publishing Co., Amsterdam-New York-Oxford, 1977. North-Holland Mathematical Library, Vol. 16.

[25] E. Năstase and P. Sissokho. The maximum size of a partial spread in a finite projective space. Journal of Combinatorial Theory, Series A, 152:353-362, 2017.

[26] B. Segre. Teoria di galois, fibrazioni proiettive e geometrie non desarguesiane. Annali di Matematica Pura ed Applicata, 64(1):1-76, 1964.

[27] V. D. Tonchev. Codes and designs. Handbook of coding theory, 2:1229-1267, 1998.

[28] S.-T. Xia and F.-W. Fu. Johnson type bounds on constant dimension codes. Designs, Codes and Cryptography, 50(2):163-172, 2009. 